

\section{PROPUESTA PARA LA IMPLEMENTACIÓN DE LA METODOLOGIA BIM EN UNA EXPERIENCIA ÁULICA ORIENTADA A LA SUSTENTABILIDAD EDILICIA}

\section{PROPOSAL FOR THE IMPLEMENTATION OF THE BIM METHODOLOGY IN AN CLASSROOM EXPERIENCE FOCUSED ON BUILDING SUSTAINABILITY}

\author{
Analia Alejandra Alvarez \\ Doctora en Arquitectura y Urbanismo \\ Jefe de trabajos prácticos Instituto de Estudios en Arquitectura \\ Ambiental - Facultad de Arquitectura, Urbanismo y Diseño - \\ Becaria Posdoctoral CONICET \\ Universidad Nacional de San Juan, San Juan, Argentina \\ https://orcid.org/0000-0003-0069-8173 \\ ana_alv023@hotmail.com
}

\author{
María Verónica Ripoll-Meyer \\ Arquitecta \\ Jefe de trabajos prácticos Instituto de Estudios en Arquitectura \\ Ambiental - Facultad de Arquitectura, Urbanismo y Diseño - \\ Becaria Posdoctoral CONICET \\ Universidad Nacional de San Juan, San Juan, Argentina \\ https://orcid.org/0000-0003-1757-2526 \\ veronicaripoll@yahoo.com
}

\section{RESUMEN}

Building Information Modeling (BIM) es una metodología de trabajo colaborativo que concentra toda la información de un proyecto en un único prototipo virtual susceptible de ser estudiado a lo largo de su ciclo de vida. Una de las principales dificultades para su implementación es la escasa formación de profesionales en su uso y alcance. Por ello, esta investigación surge con el objetivo de proponer una mecánica de trabajo en tiempo real que, a partir del uso de la metodología BIM desde instancias iniciales del proceso de diseño, constituya un sistema de apoyo a la toma de decisiones en relación con la sustentabilidad edilicia. Con tales fines, se establecen lineamientos básicos para el desarrollo de una experiencia áulica piloto que integre el uso de la metodología BIM, a partir de la realización del Building Energy Model (BEM) de tres prototipos de vivienda social de uso generalizado en San Juan, Argentina. Como resultado, se obtiene el BIM Execution Plan (BEP) para la primera implementación de la metodología BIM en el Taller Vertical de Arquitectura Ambiental (TVAA) de la Facultad de Arquitectura, Urbanismo y Diseño (FAUD) de la Universidad Nacional de San Juan (UNSJ).

Palabras clave

BIM, mecánica de trabajo, experiencia áulica, BEM, BEP

\section{ABSTRACT}

Building Information Modeling (BIM), is a collaborative methodology that gathers all the information of a project in a single virtual prototype, which can then be studied throughout its life cycle. One of the main difficulties in its use is the limited training of professionals in its use and scope. Consequently, this research proposes a real-time work procedure where, by using the BIM methodology in the initial stages of the design process, this constitutes a support system for decision-making with respect to Building Sustainability. With this in mind, basic guidelines are set out to develop a pilot classroom experience that integrates the use of the BIM methodology, by making a Building Energy Model (BEM) of three commonly used social housing prototypes in San Juan, Argentina. The result is the BIM Execution Plan (BEP) for the first use of the BIM methodology in the Vertical Workshop on Environmental Architecture (TVAA) of the Faculty of Architecture, Urbanism and Design (FAUD) of the National University of San Juan (UNSJ). 


\section{INTRODUCCIÓN}

El Building Information Modeling o Modelado de Información para la Construcción, BIM por sus siglas en inglés, es un conjunto de metodologías, tecnologías y estándares que permiten diseñar, construir y operar colaborativa e interdisciplinariamente una edificación o infraestructura, a lo largo de su ciclo de vida, en un espacio virtual (PlanBIM, 2019). Salinas y Prado (2019) sostienen que los referentes a nivel mundial en el desarrollo de estándares y normativas BIM son Estados Unidos, Reino Unido y Singapur. En América del Sur, Brasil y Chile son los más avanzados en la implementación BIM desde el sector público. En Argentina, el estándar BIM surgió desde el sector privado, pero con la participación del sector público. El resto de la región, presenta iniciativas individuales, sin concretar documentos que reflejen un trabajo colaborativo (Salinas y Prado, 2019).

Una de las principales dificultades detectadas en relación con la expansión BIM es la falta de profesionales capacitados. Para contrarrestar esta problemática, el rol de las universidades es fundamental (Orrego, 2017). En relación con el estado del arte, se observa que Piña, Varela, Aguilera y Vidales (2017) proponen revisar los planes de estudio para incorporar más competencias relativas a la metodología BIM. Para ello, definen un organigrama que establece el rol de los agentes BIM, en las distintas fases del proyecto (Diseño, Construcción y Explotación), el grado de implicación y nivel de conocimientos requeridos, a efectos de que los alumnos de Ingeniería de la Edificación conozcan de antemano las relaciones existentes entre dichos actores.

Así también, Meana, Bello y García (2017) y Reyes, Prieto, Cortés y Candelario (2017) analizan el uso de la metodología BIM en carreras de Ingeniería Industrial. Meana et al. (2017) plantean la necesidad de adaptar el modelo formativo actual a las necesidades de los profesionales de modelos colaborativos. En ese sentido, postula establecer una comisión interuniversitaria que direccione y unifique los objetivos y competencias en relación con dicha metodología. Reyes et al. (2017), por su parte, concluyen que la tecnología BIM puede utilizarse en docencia universitaria con altas probabilidades de éxito.

Si bien existe un amplio número de investigaciones relativas al uso de la metodología BIM en distintos campos de la industria de la construcción, no se ha abordado su implementación en prácticas pedagógicas orientadas a la sustentabilidad en la edificación desde instancias tempranas del proceso de diseño. En esta dirección, los autores mencionados no exponen el uso de documentos tales como el BIM Execution Plan (BEP), o bien, la elaboración de un Building Energy Model (BEM). Asimismo, el desarrollo de estándares relativos a la metodología BIM se corresponde con su aplicación en el sector público. Sin embargo, no hay estándares que orienten la forma en que dicha metodología debe ser implementada en el ámbito académico.

González Pérez (2015) señala que el estándar BIM es un marco común que se debe realizar en la fase previa a iniciar el proyecto e influye en el correcto funcionamiento del trabajo colaborativo. En PlanBIM (2019) se indica que un estándar es un documento establecido por consenso y aprobado por un organismo reconocido que se entrega para ayudar a obtener un grado óptimo de ordenamiento en un contexto dado.

Por otro lado, Chong y Wang (2016), Sakin y Kiroglu (2017) y Chaves, Tzortzopoulos, Formoso y Shigaki (2015) coinciden en que BIM constituye una base confiable para tomar decisiones conducentes a la incorporación de enfoques sostenibles y al mejoramiento del rendimiento edilicio al permitir monitorear un proyecto a lo largo de su ciclo de vida. Mercader Moyano Camporeale y Cózar-Cózar (2019) destacan que la consideración de los problemas ambientales durante la etapa de diseño representa uno de los mayores desafíos para los diseñadores.

Sobre dicha base, el presente artículo surge con el objetivo de proponer una mecánica de trabajo en tiempo real que, a partir del uso de la metodología BIM desde instancias iniciales del proceso de diseño, constituya un sistema de apoyo a la toma de decisiones en relación con la sustentabilidad edilicia. Desde esa perspectiva, inicialmente se definen lineamientos básicos para la realización de un BEM orientado al análisis del nivel de confort de la vivienda social. Para ello, se toman como punto de partida las características térmicas de los materiales que integran las soluciones constructivas adoptadas en tres prototipos de vivienda de interés social de aplicación generalizada en la ciudad de San Juan ${ }^{1}$, Argentina. De igual modo, se sientan las bases para la diagramación de un BIM Execution Plan (BEP) dirigido a la primera implementación de la metodología BIM en una experiencia áulica que tendrá lugar en agosto de 2020, en el Taller Vertical de Arquitectura Ambiental (TVAA) de la Facultad de Arquitectura, Urbanismo y Diseño (FAUD) de la Universidad Nacional de San Juan (UNSJ), con lo cual se aporta al desarrollo de futuros estándares BIM en educación. En suma, se pretende contribuir a la sustentabilidad del hábitat concientizando a los futuros profesionales respecto de la importancia que las decisiones de proyecto tienen a lo largo del ciclo de vida del edificio. Al respecto, precisar la mecánica de trabajo 
necesaria para pasar del BIM al BEM permite redefinir y mejorar, en la etapa previa a la ejecución de la obra, aquellos aspectos tecnológicos, constructivos y de diseño que aporten a una mayor sustentabilidad en la edificación.

\section{METODOLOGÍA}

Este artículo propone un conjunto de documentos capaces de estructurar una futura práctica pedagógica que, con base en la metodología BIM, favorezca la integración de variables de sustentabilidad edilicia en el proceso de diseño de los alumnos de Arquitectura. A partir de ello, podrán definirse las directrices básicas para la elaboración de un Estándar orientado a la implementación de la metodología BIM en la enseñanza de la Arquitectura en general y de la sustentabilidad edilicia en particular.

Desde este enfoque, la metodología propuesta se sustenta en dos etapas sucesivas. En la primera de ellas se definen los lineamientos básicos para el desarrollo del BEM, luego de analizar el nivel de confort de tres prototipos de vivienda de interés social de aplicación generalizada en la ciudad de San Juan, Argentina. Aquí, es importante considerar que, si bien EnergyPlus constituye un referente del análisis realizado, otros softwares para el modelado de información edilicia, tales como Revit y ArchiCAD, tienen mayor facilidad de uso, lo cual potencia su aplicación para la valoración en etapas tempranas de diseño. El desarrollo de esta etapa incluye la determinación de la mecánica de trabajo necesaria para pasar del BIM al BEM, así como también se mencionan generalidades relativas al Manual Básico de Entrega de Información (MEI) y se determina la importancia de las Solicitudes de Información BIM (SDI BIM). Adicionalmente, se analiza el contenido de los informes obtenidos de la evaluación, es decir, las variables de salida posibles en relación con los datos ingresados en el BEM. De esta instancia surgen las similitudes y divergencias asociadas al uso de cada software. Esto resulta de interés a fin de que el docente pueda orientar al alumno en la correcta interpretación de los resultados y, en consecuencia, en la determinación de estrategias de diseño bioclimático que aporten a mejorar el nivel de confort obtenidos en la simulación. Los contenidos de cada informe de evaluación se analizan conforme a seis variables (transparencia, trazabilidad, procesamiento, comparabilidad, complejidad y variables de salida posibles).

En la segunda etapa, se establecen lineamientos generales para la implementación áulica de la metodología BIM. Con tal objetivo, se propone un workflow destinado a la incorporación de variables de sustentabilidad en instancias iniciales del proceso de diseño. Por último, se construye una matriz de roles y el PEB de Oferta que direcciona el desarrollo de la práctica pedagógica. La Figura 1 sintetiza la metodología de investigación propuesta.

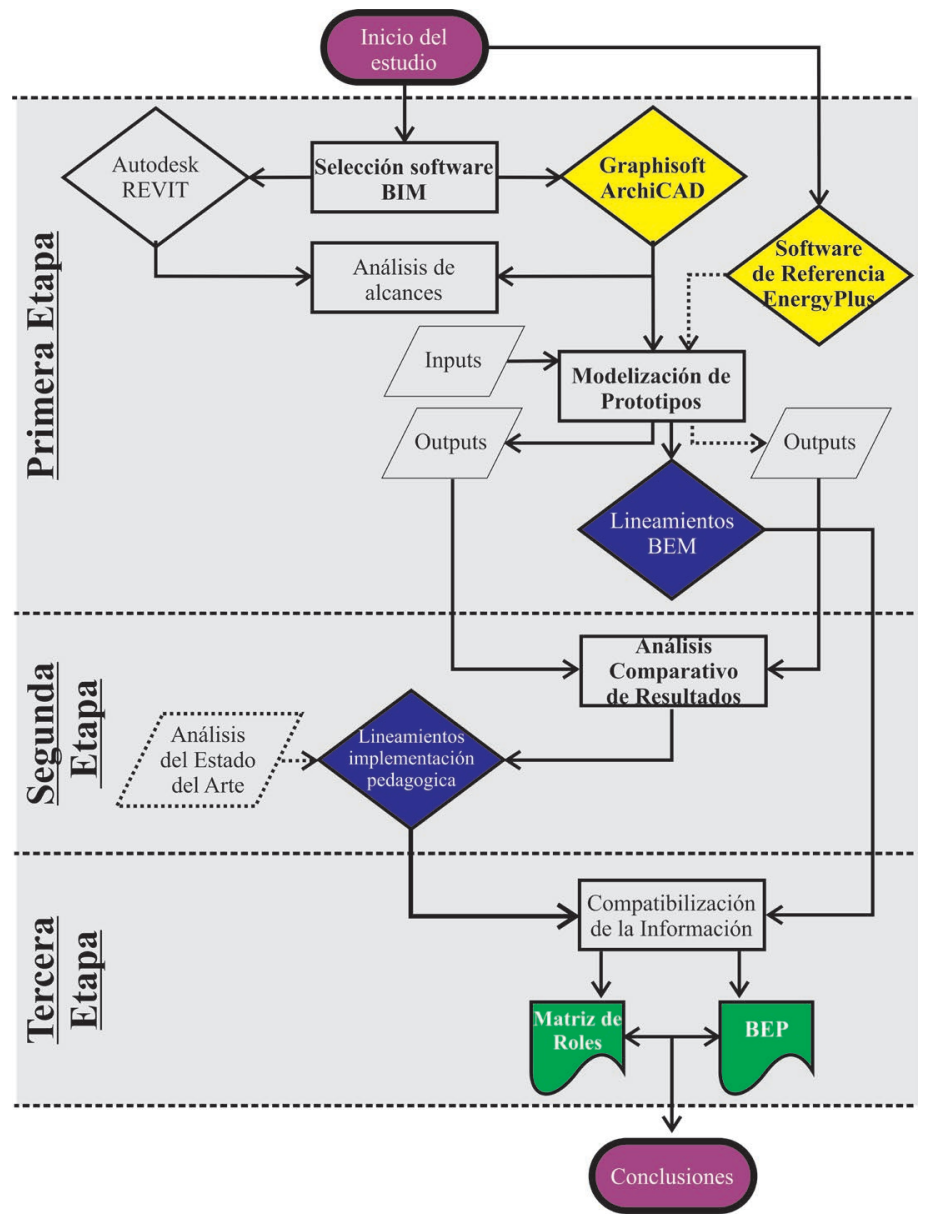

Fuente: Elaboración de las autoras.

Figura 1. Flowsheet de la metodología de investigación propuesta.

\section{ETAPA 1: LINEAMIENTOS PARA EL BEM}

Tanto para Lu, Wub, Changa y Lib (2017) como para Martin, Franco, Broock, González y Assef (2014), los métodos tradicionales de diseño son limitados en términos de análisis de sustentabilidad como resultado de la fragmentación de la información manejada por los mismos. Por ello, incluir la evaluación de energía en el flujo de trabajo del diseño arquitectónico facilita la creación de proyectos que cumplan e, incluso, superen las normativas de eficiencia energética (Soltani, 2016). No obstante, generar un modelo paramétrico requiere habilidades de manejo de software que van más allá de la representación tridimensional e implica conocer la forma en que deben asignarse los parámetros para construir un modelo BIM funcional y útil (Mojica, Valencia, Gómez y Alvarado, 2016).

El término BEM se refiere a una herramienta de simulación para el cálculo de la carga térmica y la utilización de energía en edificios que permite predecir su uso con base en la arquitectura y los sistemas de ventilación, calefacción y aire acondicionado (Jiménez, Sarmiento, Gómez y Leal, 2017). Por tanto, para realizar una evaluación energética correcta, el modelo 3D del edificio tiene que contener al menos las estructuras envolventes y la carpintería, así como 
todas las estructuras internas principales que representen un volumen de almacenamiento de calor significativo (Graphisoft, 2017).

De acuerdo con Llave Zarzuela, Arco Díaz e Hidalgo García (2019), a nivel mundial, Revit y ArchiCAD son las herramientas de modelado de información edilicia que incluyen evaluación energética más usadas por los profesionales de la construcción. Indican, igualmente, que la herramienta de evaluación energética integrada de Revit es insight 360 y la de ArchiCAD es Ecodesigner. Además, mencionan que insight 360 es un motor analítico que no está suficientemente testado, no obstante, se puede utilizar la herramienta Green Building Studio. Por último, destacan que Ecodesigner es considerado uno de los softwares de simulación energética más exactos, ya que presenta un rango de error menor al $5 \%$ en las evaluaciones de rendimiento energético (Llave Zarzuela et al., 2019). Blat Tatay (2016), en tanto, caracteriza los softwares mencionados a partir de sus principales cualidades y concluye que Revit y ArchiCAD son herramientas potentes y equivalentes que han alcanzado la madurez suficiente para ser representativos del momento que vive la metodología BIM.

En consideración con los objetivos propuestos para esta investigación, es necesario destacar la importancia del uso de herramientas que permitan la valoración de la sustentabilidad edilicia desde instancias iniciales del proceso de diseño. En esa línea, resulta relevante que los softwares a utilizar en el desarrollo de la experiencia áulica sean fáciles de usar por parte de los alumnos. Sobre dicha base, y con miras a la determinación de los lineamientos básicos para el desarrollo del BEM, se propone utilizar los softwares Revit, ArchiCAD y EnergyPLus a fin de obtener sus similitudes, divergencias y alcances, tanto a nivel de uso/interfaz como de variables de salida.

Para la realización de los modelos se utilizó ArchiCAD, versión 21 (demo), Revit 18 (demo) y EnergyPlus 8.4.0. Los prototipos modelizados son el A-13, B-13 y A-12 (Figuras 2, 3 y 4). La información gráfica fue provista por el Instituto Provincial de la Vivienda de San Juan (IPV-San Juan). En todos los casos, los prototipos se consideran ubicados en un lote con orientación norte-sur con acceso principal por la cara sur del mismo. Esto último constituye una simplificación que permite contemplar los resultados conforme a las mismas condiciones de ubicación. La Tabla 1 sintetiza las características térmicas de los materiales usados para la construcción de las viviendas.

\section{ETAPA 2: LINEAMIENTOS PARA PRÁCTICA PEDAGÓGICA}

Blasco Gutiérrez, Parant, Olivier, González Redondo y García (2017) manifiestan que la metodología BIM constituye una evolución del trabajo colaborativo en todas las partes de un proyecto $y$, por ende, la enseñanza universitaria se debe adaptar al actual perfil digital del alumno a partir de
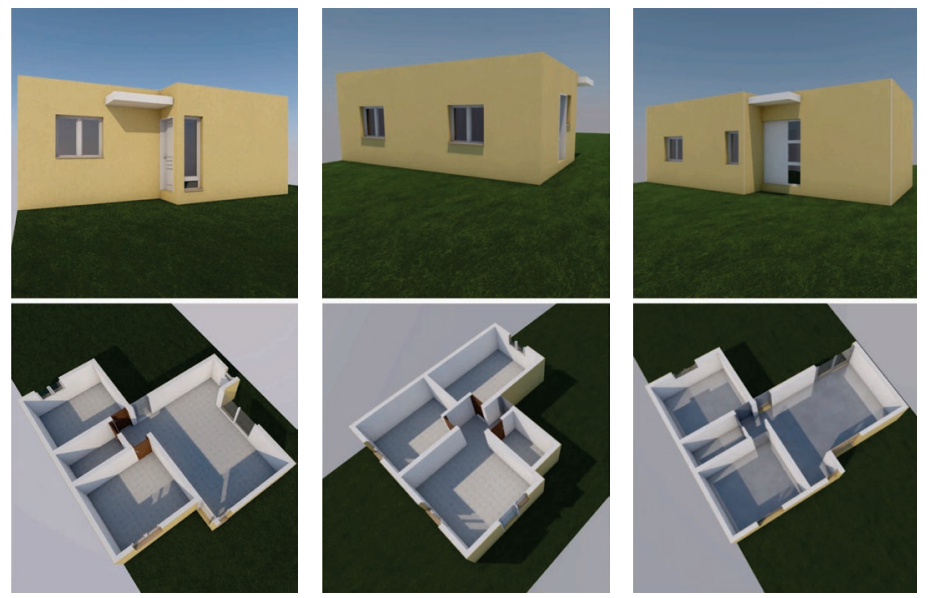

Figura 2. Prototipo A-12

Figura 3. Prototipo A-13.

Figura 4. Prototipo B-13.

Fuente: Elaboración de las autoras con base en datos del IPV-San Juan.

\begin{tabular}{|c|c|c|c|c|}
\hline \multirow{2}{*}{ Elemento } & \multirow{2}{*}{$\begin{array}{l}\text { Capas del elemento } \\
\text { constructivo }\end{array}$} & $\mathrm{R}$ & \multicolumn{2}{|c|}{$K\left(W / m^{2} K\right)$} \\
\hline & & $\left(\mathrm{m}^{2} \mathrm{~K} / \mathrm{W}\right)$ & Invierno & Verano \\
\hline \multirow{3}{*}{$\begin{array}{c}\text { Muros } \\
\text { Exteriores }\end{array}$} & Mortero & 0.01 & & \\
\hline & Ladrillón & 0.22 & 2.374 & 2.374 \\
\hline & Mortero & 0.02 & & \\
\hline \multirow{6}{*}{$\begin{array}{c}\text { Muros } \\
\text { Interiores }\end{array}$} & Mortero & 0.02 & & \\
\hline & Ladrillón & 0.22 & 2.36 & 2.36 \\
\hline & Mortero & 0.02 & & \\
\hline & Mortero & 0.02 & & \\
\hline & Ladrillón & 0.09 & 3.46 & 3.46 \\
\hline & Mortero & 0.02 & & \\
\hline \multirow{5}{*}{ Techo } & Membrana Asfáltica & 0.01 & & \\
\hline & Carpeta de Nivelación & 0.04 & & \\
\hline & Pomeca (Piedra pómez natural) & 1.25 & 0.647 & 0.619 \\
\hline & Losa Hormigón Armado & 0.09 & & \\
\hline & Cielorraso a la Cal & 0.02 & & \\
\hline \multirow{3}{*}{ Piso } & Contrapiso & 0.1 & & \\
\hline & Carpeta & 0.03 & 3.29 & 2.67 \\
\hline & Cerámico & 0.03 & & \\
\hline $\begin{array}{c}\text { Puertas } \\
\text { Exteriores }\end{array}$ & $\begin{array}{l}\text { MDF } 18 \mathrm{~mm} \\
\text { Aire } \\
\text { MDF } 18 \mathrm{~mm}\end{array}$ & \multicolumn{3}{|c|}{ Se adopta: 3.50} \\
\hline $\begin{array}{c}\text { Puertas } \\
\text { Interiores }\end{array}$ & $\begin{array}{l}\text { MDF } 5 \mathrm{~mm} \\
\text { Aire } \\
\text { MDF } 5 \mathrm{~mm}\end{array}$ & \multicolumn{3}{|c|}{ Se adopta: 3.50} \\
\hline Ventanas & Vidrio Incoloro $3 \mathrm{~mm}$ & \multicolumn{3}{|c|}{ Se adopta: 5.82} \\
\hline
\end{tabular}

Tabla 1. Características Térmicas (Resistencia y Transmitancia) de los Materiales empleados en las viviendas analizadas. Fuente: Elaboración de las autoras.

Referencias: Niveles de confort en W/m²K de la IRAM 11605 (Zona Bioambiental III)

Condición de verano: A (Muros: 0.50 Techo: 0.19) a (Muros: 1.25 Techo: 0.48) —C (Muros: 2.00 Techo: 0.76) $\square$ No verifica Condición de Invierno: A (Muros: 0.286 Techo: 0.246) B (Muros: 0.758 Techo: 0.642$) \square C$ (Muros: 1.31 Techo: 1.00$) \square$ No verifica 
ayudar a los mismos a distinguir los diferentes roles de su profesión, desde el inicio. En otras palabras, la educación BIM debe centrarse en mejorar el flujo de comunicación y la secuencia de trabajo (Latorre, Sanz y Sánchez, 2019). Para Granero y García Alvarado (2014), la enseñanza de la arquitectura contempla una dedicación fundamental a los talleres de diseño. Por consiguiente, la implementación BIM encuentra en dichos espacios curriculares las condiciones propicias para su desarrollo.

En este marco, para Piña et al. (2017), llevar a cabo una práctica pedagógica con base en la metodología BIM requiere identificar las distintas fases del proyecto BIM de manera sencilla. Dichas instancias se pueden sintetizar en: Diseño, Construcción y Explotación. Reyes et al. (2017) implementan un Dispositivo Experimental que, a partir del trabajo grupal, la determinación de roles, condicionantes y normativas a cumplir, permite a los alumnos desarrollar competencias que facilitan su integración en el mundo laboral.

Asimismo, la Cámara Argentina de la Construcción (CAMARCO, 2020) señala que la implementación BIM requiere planificar cómo se realizará la transición en la forma de trabajo, así como también elegir y desarrollar un proyecto piloto. Conjuntamente, se destaca que dicho proyecto debe ser de pequeña escala, complejidad media y responder a una tipología constructiva que el equipo de trabajo domine.

En concreto, el BEP es el documento en el que convergen las estrategias, procesos, recursos, técnicas, herramientas, sistemas, etc., que se deben aplicar para asegurar el cumplimiento de los requisitos BIM solicitados por el cliente en un proyecto determinado, conforme a las fases del ciclo de vida vinculadas al mismo (ESBIM, 2018). De acuerdo con PlanBIM (2019), deben desarrollarse dos BEP: uno de Oferta (Licitación) y otro Definitivo (Proveedor Adjudicado). La diferencia entre ambos radica en el nivel de detalle de la información contenida en cada uno. El BEP de Oferta contiene información básica del proyecto, objetivos y usos del BIM, infraestructura tecnológica y competencias del equipo, entregables generales y estrategia general de colaboración. Además de una mayor especificidad en dicha información, el BEP Definitivo incluye los estándares y convenciones a utilizar.

En cuanto a la determinación de los roles de los distintos agentes que participan, Piña et al. (2017) identifican los siguientes agentes intervinientes en los proyectos BIM:

- BIM Manager: responsable de gestionar el equipo y de desarrollar y aplicar el BEP.

- BIM Coordinator: parte ejecutiva del BIM Manager, también responsable de desarrollar, aplicar y gestionar el PEB de un proyecto.

- BIM Modelator: encargado de realizar el modelado del proyecto.
- BIM Operator: encargado de la gestión de los entregables y del intercambio de archivos.

- BIM Analyst: encargado de efectuar simulaciones y análisis de los modelos BIM.

- Content Manager: lleva a cabo tareas de gestión de la información que contiene el modelo.

- Facility Manager: realiza la gestión del edificio en fase de explotación y mantenimiento en un entorno BIM.

De esta manera, una matriz de roles acorde a las características de una implementación áulica de la metodología BIM debe compatibilizar los roles definidos por los estándares BIM con las funciones, características y capacidades de docentes y los alumnos en particular. Cabe agregar que, como resultado de sus características principales, es factible desarrollar un BEP de Oferta que permita estructurar la práctica pedagógica; en tanto el BEP Definitivo surgirá en relación con la presentación final del proyecto pudiendo contener indicadores que faciliten la evaluación del mismo en función del porcentaje de cumplimento de los objetivos propuestos.

Conforme a lo antedicho, se propone realizar, en agosto de 2020, una experiencia áulica de implementación BIM en la cátedra Taller Vertical de Arquitectura Ambiental de la FAUD-UNSJ que, con base en lo expuesto por la CAMARCO (2020), consistirá en el desarrollo de una vivienda de interés social (tipología conocida por los alumnos, de pequeña escala y complejidad media). Dado que el análisis se orienta a la sustentabilidad edilicia, se considera pertinente que los alumnos que integren el equipo de trabajo del proyecto piloto posean conocimientos previos en la temática. Por esta razón, resulta oportuno que la experiencia se realice con alumnos avanzados. Adicionalmente, para la selección del equipo de trabajo se plantea la ejecución de una encuesta previa orientada a determinar el nivel de conocimientos en relación con la metodología BIM y la sustentabilidad edilicia de los alumnos del taller.

\section{RESULTADOS Y DISCUSIÓN}

La primera etapa de desarrollo de la investigación consiste en la realización de los modelos BEM correspondientes a los casos de estudios seleccionados. Los distintos prototipos fueron elaborados conforme a las soluciones constructivas de uso generalizado en las viviendas del IPV-San Juan que, como se observa en la Tabla 1, no verifican al nivel C (mínimo) establecido para la Zona Bioambiental a la que pertenece San Juan (IIla), de acuerdo con la norma IRAM 11605. Por tanto, desde una perspectiva centrada en el confort higrotérmico, dichas soluciones constructivas deben ser revisadas a efectos de dar respuesta a la normativa vigente en Argentina, en general, y a la sustentabilidad edilicia, en particular.

Sin embargo, la importancia de realizar dichos modelos en Revit, ArchiCAD y EnergyPlus, reside en que de esa tarea se desprenden los lineamientos básicos que permiten el paso del modelo BIM al BEM, así como también los alcances 
asociados a cada software.

En tal sentido, de acuerdo con PlanBIM (2019), el Manual Básico de Entrega de Información (MEI) o Information Delivery Manual (IDM) constituye una guía para la elaboración de los modelos BIM. Es decir, estructura la información para garantizar entregables BIM de calidad y asegurar la disponibilidad y posible reutilización de la información. En esta dirección, el desarrollo del MEl implica:

- Mismo lenguaje, a fin de eliminar tareas ineficientes.

- OpenBIM IFC.

- Misma estructura. Se relaciona con la sistematización y codificación de la información (denominación coherente y uniforme de los archivos, posición coordinada, coincidencia de los nombres correspondientes a los niveles del modelo BIM, así como también el uso correcto de entidades).

- Disponibilidad de la información para usos futuros, como resultado del adecuado uso de las propiedades y conjunto de propiedades definidas en IFC.

Hay que subrayar que la importancia del uso del MEl se encuentra en las posibilidades de mejorar la interoperabilidad, de aumentar la eficacia en el desarrollo de las tareas, de facilitar la sistematización de la información y, por ende, la disponibilidad de la misma para futuros usos.

Por otro lado, las Tablas 2 y 3 exponen las diferencias y similitudes detectadas en los informes de evaluación obtenidos con cada uno de los softwares utilizados en la investigación. Al respecto, se destaca que, en la Tabla 2, se cualifica el contenido de dichos informes conforme a una escala de alta, media y baja, según sea la transparencia y trazabilidad de los resultados obtenidos en los mismos y la posibilidad de realizar análisis relacionales posteriores. Con base en los objetivos de esta investigación, también se analiza la complejidad en la interpretación de los resultados y la posibilidad de establecer edificios de referencia que favorezcan la valoración de condiciones de sustentabilidad que, a su vez, sirvan de base para la determinación de indicadores de cumplimiento relativos a la práctica pedagógica. Finalmente, se toma en consideración las variables de salida posibles en cada software. La Tabla 3 muestra, además, un listado de los contenidos observados en los informes de evaluación obtenidos.

Del examen de las Tablas 2 y 3, se obtiene que ArchiCAD pone énfasis en la transparencia de los datos, al referenciar al origen de los mismos. De igual forma, los softwares estudiados presentan la posibilidad de hacer análisis comparativos de diferentes opciones constructivas o de diseño. Revit acentúa los aspectos económicos, el uso de energía fotovoltaica y el análisis por orientaciones. Sobre dicha base, se infiere que ese software pone en valor variables de diseño bioclimático. Por otro lado, EnergyPlus y ArchiCAD exponen los resultados de distintas variables sin establecer jerarquías, a partir de lo cual se deja a criterio del investigador el uso y establecimientos de prioridades de los datos. Revit presenta, por lo demás, un análisis fuertemente orientado a los costos energéticos,

\begin{tabular}{ll}
\multicolumn{1}{c}{ Variable de Análisis } & \\
\hline Transparencia & Srazabilidad
\end{tabular}

Tabla 2. Análisis del contenido de los informes de evaluación (características generales).

Fuente: Elaboración de las autoras con base en las simulaciones realizadas en Revit, ArchiCAD y EnergyPlus.

Referencias: Alta Media $\square$ Baja

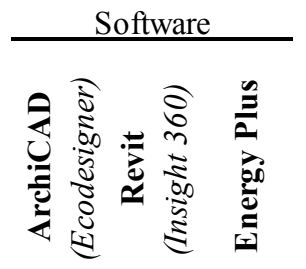

\begin{tabular}{l}
\hline Resumen Datos de Proyecto \\
Resultados por zonas \\
Balance energético del proyecto \\
Temperatura \\
Rendimiento HVAC \\
Consumo de Energía \\
Consumo de Energía por fuentes \\
Impacto Ambiental \\
Certificaciones energéticas \\
Rendimiento Base \\
Coste de energía base \\
Clasificación del rendimiento \\
Consumo y ahorros de energía \\
Infiltraciones \\
Uso de energías renovables \\
Iluminación \\
Informes de alerta
\end{tabular}

Tabla 3. Análisis del contenido de los informes de la evaluación (características específicas).

Fuente: Elaboración de las autoras con base en las simulaciones realizadas en Revit, ArchiCAD y EnergyPlus.

mientras ArchiCAD muestra una evaluación análoga a la de EnergyPlus. Asimismo, de acuerdo con la bibliografía consultada, Revit no aborda el análisis de puentes térmicos. Según lo anterior, la Tabla 4 sintetiza los lineamientos generales que permiten pasar del modelo BIM al BEM. 


\begin{tabular}{|c|c|c|c|c|c|}
\hline 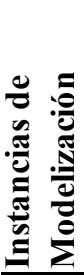 & Ítem & 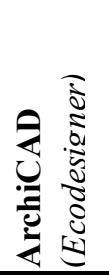 & 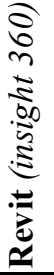 & 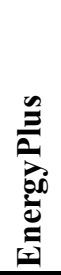 & Observaciones \\
\hline 1 & Modelado 3D integrado & $\bullet$ & $\bullet$ & - & $\begin{array}{l}\text { EnergyPlus utiliza SketchUp y } \\
\text { OpenStudio. }\end{array}$ \\
\hline 2 & $\begin{array}{l}\text { Determinación de zonas } \\
\text { térmicas }\end{array}$ & $\bullet$ & -- & $\bullet$ & \multirow{4}{*}{$\begin{array}{l}\text { ArchiCAD y Revit poseen motores de } \\
\text { cálculo integrados que toman los datos } \\
\text { del modelo. EnergyPlus utiliza } \\
\text { IDFEditor. }\end{array}$} \\
\hline \multirow{3}{*}{3} & Definición de los materiales & $\bullet$ & 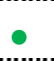 & 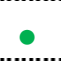 & \\
\hline & $\begin{array}{l}\text { Determinación de paquetes } \\
\text { constructivos }\end{array}$ & $\bullet$ & $\bullet$ & - & \\
\hline & $\begin{array}{l}\text { Configuración de bloques } \\
\text { térmicos / Sistemas de } \\
\text { Climatización }\end{array}$ & $\bullet$ & $\bullet$ & $\bullet$ & \\
\hline 4 & $\begin{array}{l}\text { Perfiles de uso } \\
\text { personalizados }\end{array}$ & $\bullet$ & - & - & $\begin{array}{l}\text { Revit posee perfiles de uso por } \\
\text { defecto. }\end{array}$ \\
\hline
\end{tabular}

ArchiCAD y EnergyPlus admiten

Selección de archivo _ _ _ _ archivos climáticos: $(*$.epw). Revit climático

obtiene los datos de la estación meteorológica más cercana.

En EnergyPlus, los "fatal errors" requieren la revisión del modelo. En Revit, se debe iniciar sesión en Autodesk y estar en la ventana 3D. ArchiCAD, solicita la corrección de Variables de salida inconsistencias antes de simular.

EnergyPlus y ArchiCAD, poseen variables de salida compatibles. Revit realiza un análisis ligado a la eficiencia energética y el uso de energías renovables.

\begin{tabular}{lll}
$\begin{array}{l}\text { Resultados para posterior } \\
\text { procesamiento }\end{array}$ & $-\quad-\begin{array}{l}\text { ArchiCAD y EnergyPlus permiten el } \\
\text { procesamiento posterior de los datos. } \\
\text { Informe de Resultados }\end{array}-\begin{array}{l}\text { Revit, no facilita el análisis posterior de } \\
\text { los resultados. }\end{array}$ \\
\hline
\end{tabular}

Tabla 4. Similitudes y diferencias detectadas en las distintas instancias de modelización, de los softwares analizados.

Fuente: Elaboración de las autoras con base en las simulaciones realizadas en Revit, ArchiCAD y EnergyPlus.

Del análisis de la Tabla 4, se desprenden los lineamientos guías para el desarrollo del BEM, así como las particularidades inherentes al proceso de realización del mismo, según las características del software seleccionado para la tarea. En este contexto, resulta relevante establecer claramente el objetivo para el cual se elabora el modelo. Así, si la valoración de la sustentabilidad edilicia surge en la etapa de diseño o, como en el caso de esta investigación, para constituir un sistema de apoyo a la toma de decisión desde instancias iniciales del proceso de proyectación, resulta conveniente el uso de Revit o ArchiCAD sobre la base de su fácil aplicación y la rapidez con la que se obtienen los resultados. Ahora bien, si el proyecto requiere un análisis profundo del comportamiento energético del edificio, se sugiere el uso de softwares especializados, como es el caso de EnergyPlus.

En relación con los resultados alcanzados en la segunda etapa, se destaca que, para Mercader Moyano et al. (2019), la sustentabilidad ambiental, social y económica tiene la etapa 


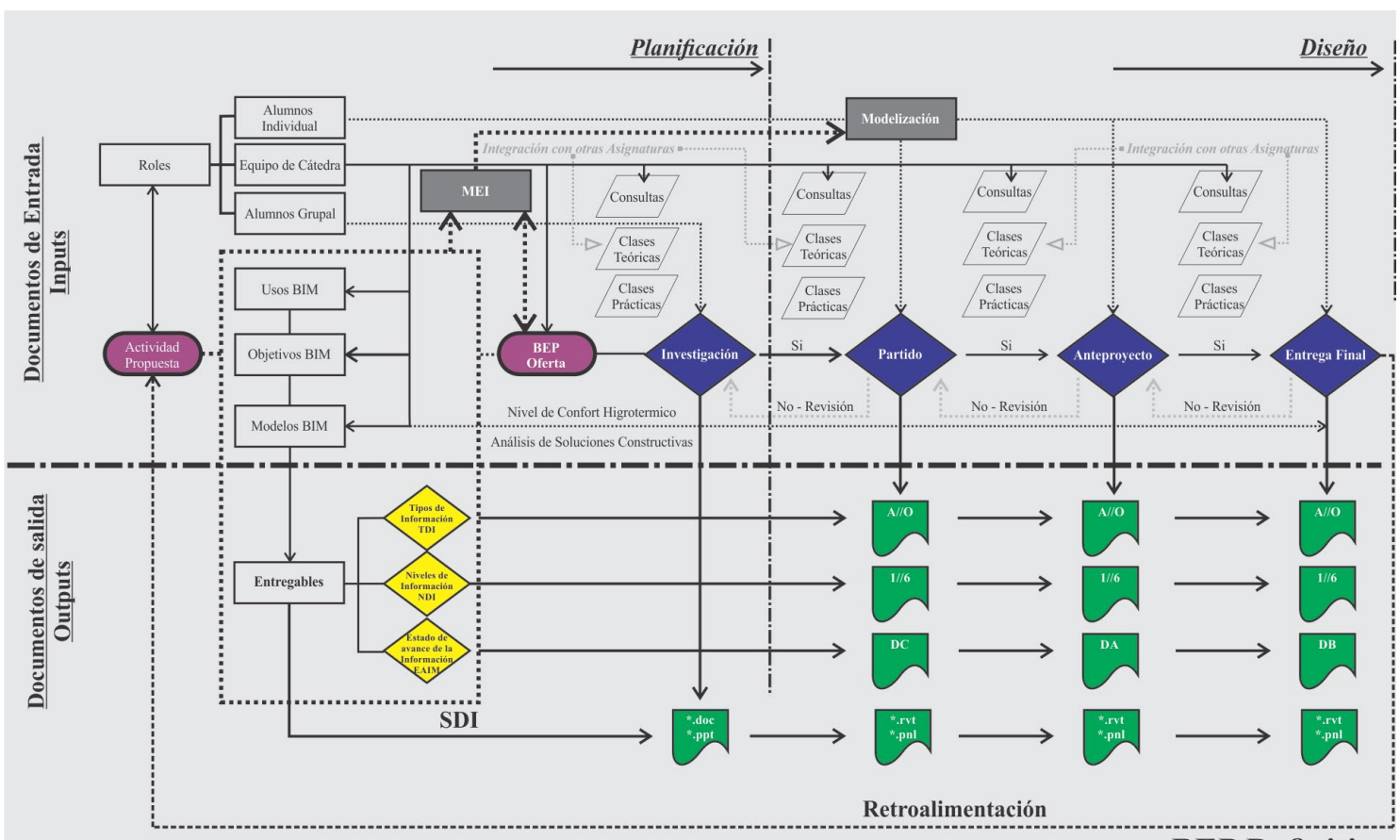

BEP Definitivo

Figura 5. Workflow Experiencia áulica con base en la metodología BIM.

Fuente: Elaboración de las autoras.

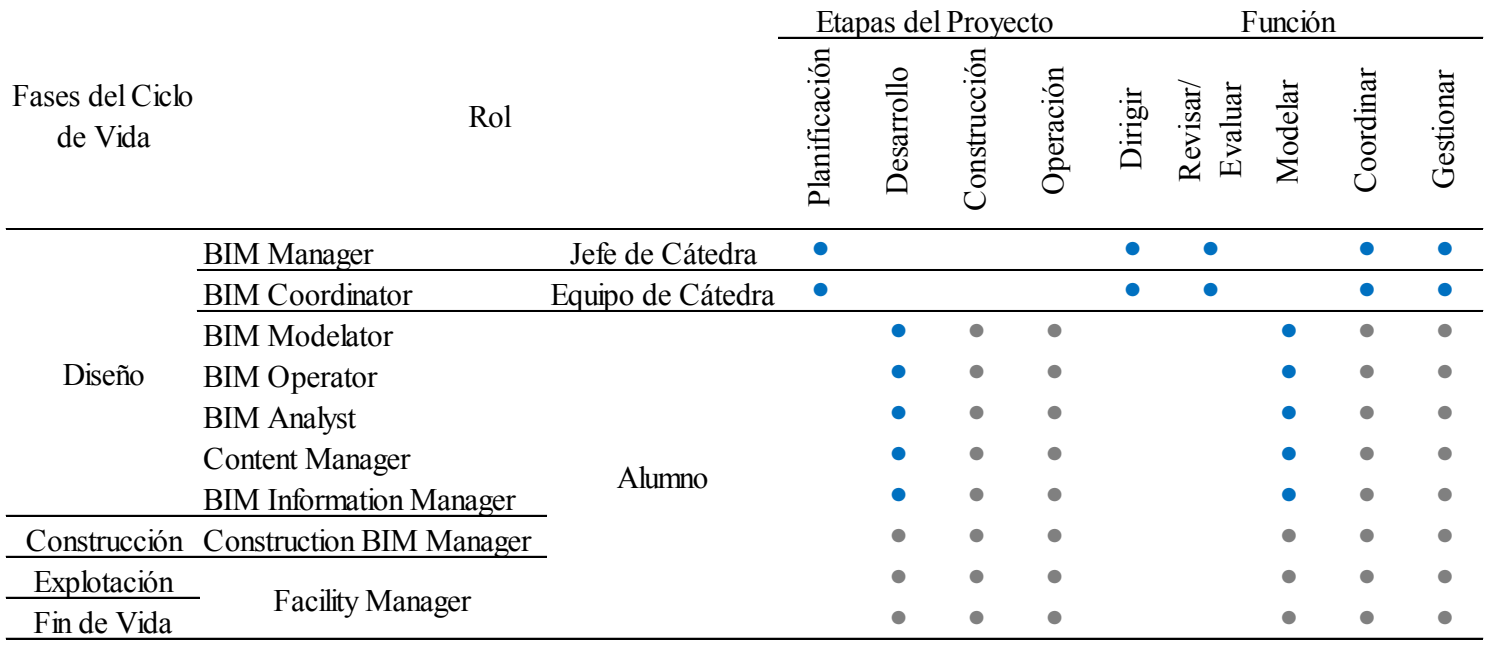

Tabla 5. Matriz tentativa de roles para la implementación áulica de la metodología BIM

Fuente: Elaboración de las autoras con base en Piña et al. (2017).

Referencias:

Rol principal

- Rol sujeto al objetivo del modelo

de diseño como núcleo. Por ello, la práctica pedagógica propuesta para ser desarrollada durante agosto de 2020, en Taller Vertical de Arquitectura Ambiental (TVAA), toma como punto de partida la fase de diseño de una vivienda de interés social. El objetivo es aquí el de proporcionar a los alumnos conocimientos que les permitan plantear un diseño sustentable a partir de la verificación de los resultados, desde el comienzo de la experiencia áulica.

Desde ese punto de vista, además de la mecánica de trabajo y el MEI, se plantean los siguientes lineamientos pedagógicos generales: conformación de grupos de trabajo; realización del diseño sustentable de una vivienda social (experiencia simplificada de diseño) a partir del uso de un software BIM; brainstorming; comparación de la experiencia con la práctica tradicional de diseño; determinación de beneficios y dificultades en la implantación de la metodología BIM durante el proceso de diseño; y elaboración de conclusiones grupales.

Es pertinente mencionar que las Solicitudes de Información BIM (SDI BIM), conocidas en los estándares europeos 


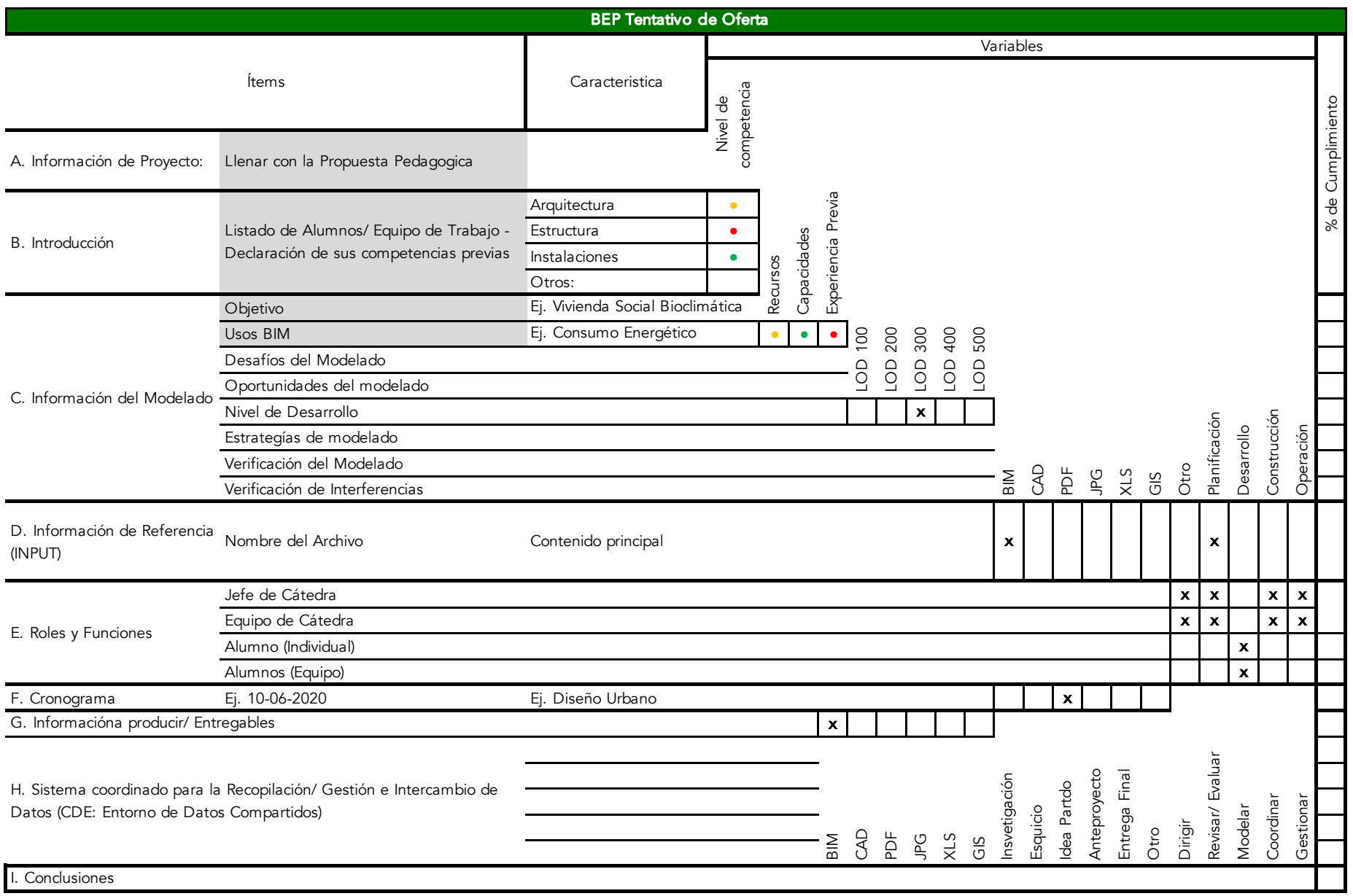

Tabla 6. BEP Tentativo de Oferta.

Fuente: Elaboración de las autoras con base en PlanBIM (2019) y SIBIM (2019).

como EIR (Employeer's Information Requirements), son documentos que incorporan: objetivos, usos, tipo y niveles de información (TDI y NDI), entregables, estrategias de colaboración (Entorno de Datos Compartidos - CDE), estado de avance de la información (EAIM) y organización de los modelos (PlanBIM, 2019). Consecuentemente, como instancia previa a la elaboración del BEP, es conveniente su definición.

Teniendo en cuenta lo mencionado, se elabora la Figura 5, que sintetiza el flujo de trabajo propuesto para el desarrollo de una experiencia áulica con base en la metodología BIM. De su análisis se infiere que el desarrollo de dicha experiencia requiere la determinación de los roles a cumplir por cada uno de los actores que intervienen en el proceso de diseño (Tabla 5), así como también los documentos previos a la presentación del BEP de Oferta (Tabla 6) y posterior BEP Definitivo. Adicionalmente, expone la complejidad inherente a la implementación de la metodología BIM en una práctica pedagógica, dado que la misma requiere del establecimiento previo de documentos que estructuren su desarrollo. En este sentido, es esencial el trabajo colaborativo del equipo de cátedra y del equipo de trabajo representado por los alumnos.
La estructura principal del BEP tentativo de Oferta propuesto en esta investigación (Tabla 6) fue elaborada con base en los BEP para el sector público de los estándares BIM, desarrollados por SIBIM (2019) y PlanBIM (2019). Los ítems indicados en rojo se corresponden con los aportados que deben ser completados conforme las características de la práctica académica a implementarse. Dado que es una tabla de doble entrada, las columnas correspondientes a "Variables" indican el grado de especificidad que debe alcanzar el modelo para cumplir con los objetivos planteados. Para completar dichas variables se utiliza, según corresponda, la metodología del semáforo, o bien, se indica la característica deseada para el ítem con una cruz. De acuerdo con el estado del arte, en el caso del uso de la metodología con fines pedagógicos, los indicadores empleados se corresponden con el porcentaje de cumplimiento alcanzado. En respuesta a ello, el BEP incorpora una columna que permite al BIM-Manager/Jefe de Cátedra evaluar el nivel alcanzado por el alumno o equipo de trabajo en cada ítem considerado. De tal forma, el uso de este BEP, además de contribuir a estructurar la práctica pedagógica y fomentar el trabajo colaborativo, facilita la evaluación, en tanto le otorga a la misma trazabilidad y transparencia. 
Debe subrayarse que la experiencia áulica propuesta se corresponde con una de las primeras implementaciones de la metodología BIM en la FAUD-UNSJ. Por lo tanto, los resultados de la misma aportan a la formación de profesionales con competencias relativas a la metodología de la BIM y al trabajo colaborativo, en lo que González Pérez (2015) caracteriza como Big BIM, además del manejo de herramientas BIM (Little BIM). Igualmente, al estar orientado a la sustentabilidad edilicia se contribuye al uso de la metodología como sistema de apoyo a la toma de decisiones que pongan en valor dicha variable en etapas iniciales del proceso de diseño.

Entre las principales limitaciones para la implementación de la metodología BIM en una experiencia áulica orientada a la sustentabilidad edilicia, puede mencionarse el capital humano con el que se cuenta para la realización de la misma. En efecto, la determinación del nivel de conocimientos previos que poseen los alumnos es fundamental para direccionar los objetivos del proyecto piloto y los indicadores ambientales y de cumplimiento a evaluar. Para ello, previo a la realización de la experiencia áulica, debe llevarse a cabo una encuesta que haga posible determinar dicho nivel. Es importante retroalimentar la práctica pedagógica identificando las experiencias positivas y las mejorables. De ello, se obtienen las bases para estandarizar las prácticas positivas, redefinir aquellas a mejorar y detectar desvíos o formas ineficientes de trabajo a tiempo, lo cual se traduce en la mejora continua del equipo de trabajo (CAMARCO, 2020).

\section{CONCLUSIÓNYFUTUROSTRABAJOS}

El estudio precedente permite inferir que el uso de la metodología BIM en el ámbito académico no solo es necesario para la formación de profesionales con competencias específicas en la misma, sino que también es altamente factible. El BEP propuesto, cuya estructura guarda relación con los estándares BIM desarrollados en Argentina y Chile, facilita la planificación de una experiencia pedagógica orientada a la incorporación de variables de sustentabilidad edilicia en etapas iniciales del proceso de diseño con base en el trabajo colaborativo.

Desde esta perspectiva, simular tres prototipos de vivienda social de uso generalizado en San Juan, Argentina, permitió determinar la mecánica de trabajo necesaria para pasar del BIM al BEM. De dicha tarea, se obtienen las particularidades propias del uso de un determinado software, lo cual favorece que tanto el docente como el alumno detecten rápidamente errores cometidos durante la realización del modelo. Paralelamente, conocer los alcances de los resultados obtenidos en Revit, ArchiCAD y EnergyPLUS permite al alumno realizar la actividad propuesta (proyecto piloto) en el software de su elección sin comprometer la validez de los resultados.

La matriz de roles, el BEP y el workflow propuesto generan una estructura que facilita la transición del modelo de formación actual a modelos colaborativos. Además, al poner el foco del análisis en el desarrollo del BEM, se aporta positivamente al uso de la metodología como sistema de apoyo a la toma de decisiones relativas a variables de sustentabilidad desde instancias iniciales del proceso de diseño.

En este sentido, el BEP presentado en este artículo contribuye al desarrollo de estándares específicos que direccionen la forma de implementación de la metodología BIM en ámbitos académicos que, a su vez, garanticen que el alumno, independientemente del equipo docente y el software $\mathrm{BIM}$, desarrolle competencias encaminadas a aumentar la productividad y aportar a la sustentabilidad de la industria de la construcción. De este modo, se ayuda a concientizar a los futuros profesionales respecto de la importancia que las decisiones tomadas durante el proceso de diseño tienen en el futuro comportamiento energético del proyecto, así como también en los impactos ambientales asociados al mismo, o bien, en el nivel de confort interior de sus espacios y, con ello, en la calidad de vida de sus ocupantes.

Para efectos de retroalimentación de su estructura, en la segunda etapa de esta investigación se validará la propuesta presentada en el TVAA de la FAUD-UNSJ. Asimismo, debe subrayarse que la implementación áulica de la metodología BIM, como parte activa del proceso de diseño requiere la determinación de documentos que exceden los alcances de esta primera etapa de investigación. Al respecto, y a futuro, se plantea la necesidad de abordar análisis que incluyan la determinación y caracterización del capital humano con que se cuenta, así como también la definición de los indicadores de evaluación posibles conforme los intereses y objetivos de cada cátedra de diseño, el estudio de normativo (ISO 19650), o bien, el desarrollo de documentos fuera de los mencionados en el presente artículo tales como: AIR (Asset information requirements), $\mathrm{CDE}$ (Common data environment), OIR (Organisational information requirements), PIR (Project information requirements), entre otros. Posteriormente, se debe profundizar también en el desarrollo de MEI, SDI y BEP, guías acordes al nivel académico del alumno, que integren en su estructura el intercambio de información con otras asignaturas y laboratorios. En relación con lo último, resulta de interés ahondar en la interoperabilidad entre BIM y GIS, a fin de elaborar un Modelo de Información de la Ciudad (CIM) que aporte a la toma de decisiones relativas al entorno urbano. Con ello, la práctica pedagógica se enriquecería como resultado del abordaje de conceptos de asignatura tales como "urbanismo" o "instalaciones".

\section{AGRADECIMIENTOS}

Este artículo corresponde a la primera etapa de un proyecto interno de investigación Convocatoria 2018-2019, denominado "Aplicación de herramientas BIM en el proceso de diseño bioclimático de viviendas sociales en zonas áridas", financiado por la Facultad de Arquitectura, Urbanismo y Diseño de la Universidad Nacional de San Juan y ejecutado en el Instituto de Estudios en Arquitectura Ambiental "Arq. Alberto H. Papparelli" - Resolución Nº 093/18 - CD - FAUD. 


\section{REFERENCIAS BIBLIOGRÁFICAS}

Blasco Gutiérrez, A., Parant, A., Olivier, A., González Redondo, M. y García, A. (2017). Implementación TIC en la docencia universitaria: estudio de los esfuerzos en vigas. Advances in Building Education / Innovación Educativa en Edificación, 1(1), 37-46.

Blat Tatay, D. (2016). Nuevas metodologías y tecnologías en el proceso constructivo y mantenimiento de infraestructuras y edificios singulares. Recuperado de https://repositorio.comillas. edu/xmlui/handle/11531/14566.

Cámara Argentina de la Construcción (CAMARCO) (2020). Primeros Pasos en BIM. Recuperado de http://www.camarco.org.ar/escuelade-gestion/primeros-pasos-en-bim

Chaves, F., Tzortzopoulos, P., Formoso, C. y Shigaki, J. (2015). Using 4D BIM in the Retrofit Process of Social Housing. Recuperado de http://eprints.hud.ac.uk/id/eprint/25563/1/Paper_74.v1[1].pdf

Chong, H. y Wang, X. (2016). The Outlook of Building Information Modeling for Sustainable Development. Recuperado de https://espace.curtin.edu.au/ bitstream/handle/20.500.11937/6640/239620_239620. pdf?sequence $=2$ \&isAllowed $=\mathrm{y}$

ESBIM (2018). Guía Transversal. Guía para la elaboración del Plan de Ejecución BIM. Ministerio de Fomento. Gobierno de España. Recuperado de https://www.esbim.es/wp-content/ uploads/2018/10/GUIA-ELABORACION-PLAN-DE-EJECUCIONBIM.pdf

González Pérez, C. (2015). Building Information Modeling: Metodología, aplicaciones y ventajas. Casos prácticos en gestión de proyectos. Proyecto Final de Máster en Edificación, Especialidad de Gestión. Universidad Politécnica de Valencia. Escuela Técnica Superior Ingeniería de Edificación. España. Recuperado de https:// riunet.upv.es/bitstream/handle/10251/56357/TFM\%202015\%20 CARLOS\%20GONZALEZ.pdf?sequence=1\&isAllowed=y

Granero, A. y García Alvarado, R. (2014). Aprendizaje temprano de arquitectura sustentable mediante vistas interiores graduadas. Revista Hábitat Sustentable, 4 (1), 14-24.

Graphisoft (2017). Manual de Ayuda ArchiCAD 21 - Archivos de Programa. Recuperado de www.ghaphisoft.com.

Jiménez Roberto, Y., Sarmiento, J., Gómez Cabrera, A. y Leal del Castillo, G. (2017). Análisis de sostenibilidad ambiental de edificaciones empleando metodología BIM (Building Information Modeling). Ingeniería y Competitividad, 19(1), 230 - 240.

Latorre Uriz, A., Sanz, C., Sánchez, B. (2019). Aplicación de un modelo Lean-BIM para la mejora de la productividad en redacción de proyectos de edificación. Informes de la Construcción, 71(556), e313, 1-9. https://doi.org/10.3989/ic.67222.

Llave Zarzuela, E., Arco Díaz, J. e Hidalgo García, D. (2019). Estudio comparativo-tecnologías BIM en Edificación: Arquitectura Sostenible Comparative study-BIM technologies in Building: Sustainable Architecture. Anales de Edificación, 5(3), 8-14. http:// dx.doi.org/10.20868/ade.2019.4362

Lu, Y., Wub, Z., Changa, R. y Lib, Y. (2017). Building Information Modeling (BIM) for green buildings: A critical review and future directions. Automation in Construction 83, 134-148. http://dx.doi. org/10.1016/j.autcon.2017.08.024.

Martin Dorta, N., Franco Pérez, C., Broock Hijar, D., González De Chaves y Assef, P. (2014). Análisis de la integración de la tecnología BIM y los indicadores de Sostenibilidad EDISOST. $2^{\circ}$ Congreso NacionalBIM-EUBIM2014Encuentro de UsuariosBIM-Universidad Politécnica de Valencia, Valencia. Recuperado de https://riunet. upv.es/bitstream/handle/10251/37634/EUBIM_Encuentro\%20 de\%20usuarios\%20BIM\%202014_2\%c2\%ba\%20Congreso\%20 nacional\%20BIM_6165.pdf?sequence=1\&isAllowed =y

Meana, V., Bello, A. y García, R. (2019). Análisis de la implantación de la metodología BIM en los grados de ingeniería industrial en España bajo la perspectiva de las competencias. Revista Ingeniería de Construcción RIC., 34(2), 169-180. Recuperado de https:// scielo.conicyt.cl/pdf/ric/v34n2/0718-5073-ric-34-02-169.pdf

Mercader Moyano, M., Camporeale, P. y Cózar-Cózar, E. (2019). Evaluación de impacto ambiental mediante la introducción de indicadores a un modelo BIM de vivienda social. Revista Hábitat Sustentable, 9(2), 78 -93. https://doi.org/10.22320/07190700.201 9.09.02.07

Mojica Arboleda, A., Valencia Rivera, D., Gómez Cabrera, A., Alvarado Vargas, Y. (2016). Planificación y control de proyectos aplicando "Building Information Modeling". Un estudio de caso. Ingeniería, 20(1), 34-45.

Orrego, S. (2017). Encuesta Nacional BIM 2016: adopción de Building Information Modeling en Argentina. FODECO.

Piña Ramírez, C., Varela Lujan, S., Aguilera Benito, P. y Vidales Barriguete, A. (2017). Aprendizaje de los roles de los agentes BIM en la organización de proyectos. Advances in Building Education / Innovación Educativa en Edificación, 1(1), 47-55.

PlanBIM. (2019). Estándar BIM para proyectos públicos Intercambio de Información entre Solicitante y Proveedores. Comité de Transformación Digital CORFO. Recuperado de https:// planbim.cl/estandar-bim-para-proyectos-publicos-intercambio-deinformacion-en-solicitante-y-proveedores-sebastian-manriquez/

Reyes, A., Prieto, P., Cortés, J. y Candelario, A. (2017). Aplicación de la tecnología BIM en la asignatura de proyecto del Grado de Ingeniería Industrial en la UNEX. Advances in Building Education / Innovación Educativa en Edificación, 1(1), 68-77.

Sakin, M. y Kiroglu, Y. (2017). 3D Printing of Buildings: Construction of the Sustainable Houses of the Future by BIM. Energy Procedia $134,702-711$

Salinas, J. y Prado, G. (2019). Building information modeling (BIM) to manage desing and construction phases of Peruvian public projects. Building \& Management, 3(2), 48-59. http://dx.doi. org/10.20868/bma.2019.2.3923

SIBIM. BEP BIM Execution Plan. (2019). Plan de Ejecución BIM. Versión 01. Sistema de Implementación BIM. Ministerio del Interior Obras Públicas y Vivienda. Recuperado de https://ppo.mininterior. gob.ar/SIBIM/Library/Index

Soltani, S. (2016). The Contributions of Building Information Modelling to Sustainable Construction. World Journal of Engineering and Technology, 4, 193-199. http://dx.doi. org/10.4236/wjet.2016.42018. 